\title{
GOOD RECORD KEEPING KEY TO PHASE-DOWN OF DENTAL AMALGAM
}

Dental Protection is reminding dental practices in the UK to maintain good clinical records and obtain full consent if a decision is made to use amalgam as a restorative material, ahead of changes to EU Regulations on its use.

The EU Mercury Regulation is intended to protect the environment from the adverse effects of mercury pollution. It reflects the aims of the Minamata Treaty to reduce the use of dental amalgam in the medium to long term, and to eventually phase it out altogether.

Since 1 July 2018 amalgam has been barred from use in primary teeth, children under 15 years and during pregnancy/ breastfeeding - except if deemed necessary on the ground of 'specific medical needs'. This should be interpreted as including the specific dental needs of the patient.

By 1 July next year the UK and other EU member states will be required to have a national plan on the phasing down of the use of dental amalgam.

Dental Protection advises members to take extra care in obtaining consent and record keeping, to help in defending any future claims, complaints or regulatory investigations that may arise from the use of amalgam in the restricted groups.

Dr Raj Rattan, Dental Director at Dental Protection said: 'Complaints and claims may arise despite a clinician's efforts to ensure that patients are satisfied with their treatment. Therefore, in situations such as these extra care needs to be taken.

'If it's deemed appropriate to use amalgam in a patient in one of the restricted groups, they must communicate the rationale to the patient, or the person who has parental responsibility for them, explain why the decision is in the patient's best interest, and provide information about the material risks and benefits of amalgam in that particular situation. Valid consent must then be obtained ensuring they are aware of the restriction in specific patient groups.
'In order for the patient or their guardian's consent to be valid, they should be given the opportunity and time to ask questions about the proposed treatment to make an informed shared decision.

'Once the decision has been agreed, the justification for placing the amalgam should then be recorded in the patient's clinical records, along with any discussions about the options, risks, benefits and costs as part of the consent process.

'Records should state clearly on what basis the decision to use amalgam in one of the restricted groups was taken, and that it was made with the patient or guardian's full knowledge and understanding.'

The use of amalgam is so far not restricted in patients who do not fall into the identified groups. However, patients with knowledge of the restriction may express anxieties about the use of dental amalgam in their own mouths.

In these circumstances Dental Protection advises members to discuss the position of the EU Regulation with the patient, explain the risks and benefits, discuss any alternatives and ensure proper consent is obtained before proceeding with the treatment. Details of this discussion should be recorded in the patient's records.

Dr Raj Rattan added: 'Without proper consent and comprehensive, well-kept records, it will be difficult to defend any allegation made down the line. Detailed records of treatment will influence whether a case can be defended or whether it will need to be settled.'

\section{Further reading:}

- The Control of Mercury (Enforcement) Regulations 2017 https://www.legislation. gov.uk/uksi/2017/1200/contents/made

- Minamata Convention on Mercury http://www.mercuryconvention.org/

- British Dental Association information on dental amalgam www.bda.org/ amalgam
IMPACT OF PLASTIC STRAW BAN ON ORAL HEALTH ADVICE?

According to recent government guidelines, plastic straws may be banned in the UK from as soon as 2019. This step has been taken to help preserve marine environments, but will it have an impact on the advice we give to patients?

Straws have been found to help prevent tooth decay as they lessen the contact sugary drinks have with teeth during drinking. This has led to dental professionals recommending straws to their child patients, especially considering the higher levels of childhood tooth decay seen in recent years.

So now that plastic straws might not be available in the future, what can we do to stop the ban affecting children's oral health?

The BSDHT believes we need to explore alternatives that provide the benefits of plastic straws without impacting the environment so heavily.

Straws for life, compostable straws made from plant proteins and paper straws with wax coatings are all viable options, and by recommending these to patients we can help stop the rising tide of childhood tooth decay and save the planet at the same time.

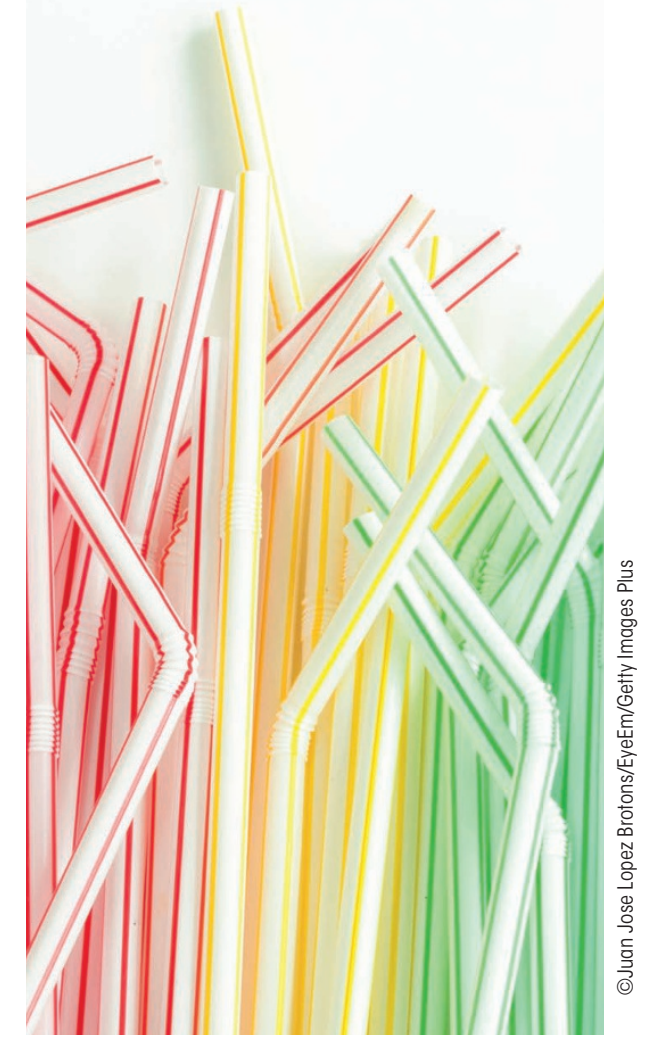

